\title{
Nebulized liposomal gadobenate dimeglumine contrast formulation for magnetic resonance imaging of larynx and trachea
}

This article was published in the following Dove Press journal:

International Journal of Nanomedicine

17 November 201।

Number of times this article has been viewed

\section{Xiaohui Wei ${ }^{1,2}$ \\ Huawei $\mathrm{Wu}^{3}$ \\ Qing $\mathrm{Lu}^{3}$ \\ Jianrong $X \mathrm{u}^{3}$ \\ Yuhong $\mathrm{Xu}^{1,2}$}

'School of Biomedical Engineering and Med-X Research Institute; ${ }^{2}$ School of Pharmacy, Shanghai Jiao Tong University; ${ }^{3}$ Department of Radiology, Renji Hospital affiliated to Shanghai Jiao Tong University, Shanghai, People's Republic of China
Correspondence: Yuhong Xu School of Biomedical Engineering and Med-X Research Institute, Shanghai Jiao Tong University, Shanghai 200030, People's Republic of China

Tel +86 2l 34204739

Fax +86 21 34204739

Email yhxu@sjtu.edu.cn

\section{jianrong Xu}

Department of Radiology, Renji Hospital affiliated to Shanghai Jiao Tong University, Shanghai 200127,

People's Republic of China

Tel +86 2I 58752345

Fax +86 2I 63736075

Email xujianr@hotmail.com
Background: To develop a lipid-stabilized contrast formulation containing gadobenate dimeglumine for clear visualization of the mucosal surfaces of the larynx and trachea for early diagnosis of disease by magnetic resonance imaging.

Methods: The contrast formulation was prepared by loading gadobenate dimeglumine into egg phosphotidylcholine, cholesterol, and sterylamine nanoliposomes using the dehydrationrehydration method. The liposomal contrast formulation was ultrasonically nebulized, and the deposition and coating pattern on explanted pig laryngeal and tracheal segments was examined by inductively coupled plasma atomic emission spectroscopy. The sizes of the nebulized droplets were characterized by photon correlation spectroscopy. The contrast-enhanced mucosal surface images of the larynx and trachea were obtained in a 3.0T magnetic resonance scanner using a T1-weighted spectral presaturation inversion recovery sequence.

Results: Various cationic liposome formulations were compared for their stabilization effects on the droplets containing gadobenate dimeglumine. The liposomes composed of egg phosphotidylcholine, cholesterol, and sterylamine in a molar ratio of 1:1:1 were found to enable the most efficient nebulization and the resulting droplet sizes were narrowly distributed. They also resulted in the most even coating on the laryngeal and tracheal lumen surfaces and produced significant contrast enhancement along the mucosal surface. Such contrast enhancement could help clearer visualization of several disease states, such as intraluminal protrusions, submucosal nodules, and craters. Conclusion: This lipid-stabilized magnetic resonance imaging contrast formulation may be useful for improving mucosal surface visualization and early diagnosis of disease originating in the mucosal surfaces of the larynx and trachea.

Keywords: lipid, gadolinium, magnetic resonance imaging, upper airway, nebulization, mucoadhesion

\section{Introduction}

There are many diseases that involve abnormalities occurring on the mucosal surfaces of the larynx and trachea. ${ }^{1}$ Among them, squamous cancer of the larynx is the most commonly occurring head and neck cancer. ${ }^{2}$ In addition, neoplasms involving the mucosal surfaces of the trachea are also abundant, but easily misdiagnosed as bronchitis or asthma because of a lack of specific symptoms. ${ }^{3}$ Endoscopic examination has been widely used to detect these mucosal abnormalities. However, disease-related submucosal spread and infiltration into the surrounding tissues are not visible at endoscopy. ${ }^{1}$ Some bulky tumors are also reported to hinder distal endoscopic visualization, particularly in the subglottic region. ${ }^{4}$ As a complementary tool, cross-sectional imaging methods, such as magnetic resonance imaging (MRI) may be used to detect abnormal soft tissue thickening and cartilage invasion or fat infiltration, and thus help in accurate depiction of the disease state. ${ }^{2,4,5}$ 
Although MRI has excellent soft tissue contrast, the imaging qualities of the lung and airway have always been poor. The low proton density of the airway and pulmonary parenchyma results in low signal-to-noise ratios. ${ }^{6}$ In addition, complex air-soft tissue interfaces produce an inhomogeneous magnetic field for imaging, and artifacts are common. ${ }^{6}$ In order to improve the quality of MRI in the airway, researchers had used hyperpolarized gas, such as ${ }^{3} \mathrm{He}^{7}$ or ${ }^{129} \mathrm{Xe},{ }^{8}$ or nebulized gadolinium solutions. ${ }^{9}$ However, these agents were designed mainly to evaluate lung ventilation and do not deposit on the lumen surfaces. Therefore, it has been difficult to visualize the mucosal surfaces using MRI. ${ }^{2}$ To address this problem, we developed a contrast formulation that can coat the mucosal surfaces of the larynx and trachea evenly, thus helping visualization and detection of small foci in early stages of disease development.

Mucoadhesive systems have been widely used for intranasal, ${ }^{10}$ buccal, ${ }^{11}$ peroral, ${ }^{12,13}$ and airway ${ }^{14,15}$ drug delivery. Longer retention of drugs adhering to mucosal surfaces has been shown to improve in situ or systemic therapeutic effects significantly. The most commonly used mucoadhesive excipients are hydrophilic macromolecules, such as carbomers ${ }^{16}$ sodium alginate, ${ }^{11}$ chitosan, ${ }^{15}$ and cellulose derivatives. ${ }^{17}$ However, these can be highly viscous and unsuitable for airway administration. In some recent research, cationic lipids were also shown to promote adhesion through interactions between cationic vehicles and the negatively charged mucosal surface. ${ }^{18}$ Lipids are also useful for modulating the aerodynamic diameters of nebulized droplets for optimal distribution in the respiratory tract and deposition on the laryngeal and tracheal surfaces. ${ }^{19}$ In this study, in order to achieve even coating of the mucosal surfaces, we included egg phosphotidylcholine and cholesterol in a formulation for stabilizing gadobenate dimeglumine droplets, and added stearylamine to improve mucoadhesiveness. The formulations were optimized to provide significant and even imaging contrast enhancement of the mucosal surfaces.

\section{Materials and methods Materials}

Egg phosphatidylcholine of $99 \%$ purity was purchased from Lipoid GmbH (Ludwigshafen, Germany), and cholesterol and stearylamine of $97 \%$ and $99 \%$ purity, respectively, were purchased from Sigma-Aldrich Co Ltd (St Louis, MO). Gadobenate dimeglumine injection (MultiHance ${ }^{\circledR}$ ) was obtained from Shanghai Bracco Sine Pharmaceutical Corporation Ltd (Shanghai, China). The other reagents used in this study were of analytical grade and purchased from Shanghai Chemical Reagent Co Ltd (Shanghai, China).

\section{Preparation of lipid-stabilized contrast formulation}

The lipid-stabilized contrast agent was prepared by lyophilization-rehydration. Egg phosphatidylcholine, sterylamine, and cholesterol in different ratios were dissolved in tert-butanol. MultiHance injection was slowly added to the lipid solution and mixed well. The resulting transparent solutions were dispersed in vials and lyophilized $(0.100 \mathrm{mbar}$, 36-48 hours). The lipid-stabilized contrast formulations were obtained by rehydration of the lyophilized powder with water for injection in $50^{\circ} \mathrm{C}$ to a concentration of $12 \mathrm{mg} \mathrm{Gd} / \mathrm{mL}$, and homogenized in an ultrasonic water bath.

\section{Measurement of particle size and zeta potential}

The particle size and zeta potential of the gadobenate dimeglumine encapsulated/complexed liposomes were measured by photon correlation spectroscopy (Zetasizer $3000 \mathrm{HSa}$, Malvern, Worcestershire, UK) at $25^{\circ} \mathrm{C}$. The samples were diluted with deionized water. The measurements were carried out three times and the results were averaged.

\section{Nebulization of lipid-stabilized contrast formulations}

The lipid-stabilized contrast formulations in $30 \mathrm{~mL}$ volume were put into an ultrasonic nebulizer (YUYUE402, Shanghai Medical Instrument Company, Shanghai, China) and nebulized using the following parameters: frequency $1.5 \mathrm{mHz}$, water bath temperature $50^{\circ} \mathrm{C}$, nebulization volume $4 \mathrm{~mL} / \mathrm{min}$, and medium airstream flow.

\section{Measurement of droplet size of nebulized contrast formulations}

The droplet sizes of the nebulized lipid-stabilized contrast formulations were measured using a Spraytec RTSizer (Malvern) at room temperature in auto mode. The measurement parameters were: path length $12.00 \mathrm{~mm}$, scattering rings 3-31, size bins $0-59$, and mesh factor 1 , with multiple scattering enabled. Diluted MultiHance injection in the same concentration (12 $\mathrm{mg} \mathrm{Gd} / \mathrm{mL}$ ) was used as the control.

\section{Preparation of explanted pig larynx and trachea segments}

Freshly explanted pig tracheas with an intact larynx were ordered from a slaughter house. The blood on the segments was carefully cleaned with cotton balls and the tracheas were cut to be around $30 \mathrm{~cm}$ in length. 
Three diseased tissue models with representative mucosal abnormalities were prepared. The intraluminal protrusion model was prepared by implanting three lean pork balls with diameters of $5 \mathrm{~mm}, 10 \mathrm{~mm}$, and $15 \mathrm{~mm}$ inside the tracheal lumen. The submucosal nodule model was prepared by injection of $1 \%$ methyl cellulose under the tracheal mucosal surface to form "lumps" of about $3 \mathrm{~mm}, 5 \mathrm{~mm}$, and $8 \mathrm{~mm}$ in diameter, respectively. For preparation of the submucosal crater lesion model, a small area of tracheal tissue was resected to about $1-2 \mathrm{~mm}$ in depth, and then the tracheal tissue was sewn up.

\section{Characterization of contrast agent deposition on luminal surface}

The lipid-stabilized contrast formulations were nebulized and delivered to the horizontally placed ex vivo pig tracheal segments from the direction of the larynx. The tracheas were stretched softly during delivery. For each trachea, nebulization delivery lasted for two minutes. Diluted MultiHance injection in the same concentration $(12 \mathrm{mg} \mathrm{Gd} / \mathrm{mL})$ was nebulized for the comparison.

After nebulization delivery, the pig tracheal segments were cut open horizontally and divided into top half and bottom half segments (Figure 1). Each half segment was further divided into three areas, based on their distances to the larynx, ie, the proximal area ( $3 \mathrm{~cm}$ to the larynx), the middle area ( $15 \mathrm{~cm}$ to the larynx), and the distal area $(27 \mathrm{~cm}$ to the larynx). For each area, a $1 \mathrm{~cm}^{2}$ trachea sample in a square shape was collected. The samples were homogenized and treated with nitric acid. Gadolinium concentrations in each tracheal sample were measured using inductively coupled plasma atomic emission spectroscopy (IRIS/AP, Thermo Fisher Scientific, Waltham, MA).

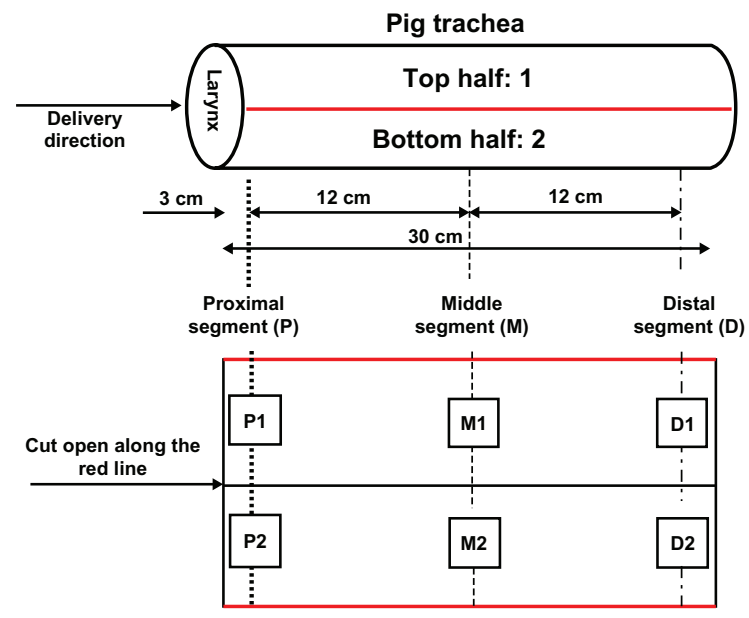

Figure I Schematic illustration of the sampling operation for the deposition study of nebulized lipid-stabilized contrast agent on the luminal surface of ex vivo pig laryngeal and tracheal segments.

\section{Magnetic resonance imaging of larynx and trachea}

The nebulized lipid-stabilized contrast formulation was delivered to the ex vivo pig tracheal segments using the same method as described earlier. The tracheal samples were numbered and enwrapped inside fresh lean meat to eliminate the interference of air on imaging. MRI examination of the tracheas before and after contrast agent delivery was performed on a Philips Intera Achieva 3.0 Tesla MR scanner (Philips, The Netherlands) with a SENSE head loop using a T1-weighted spectral presaturation inversion recovery sequence.

\section{Statistical analysis}

The data were presented as the mean \pm standard deviation. Statistical analysis was performed using the Student's $t$-test, and differences were judged to be significant at $P<0.05$.

\section{Results}

\section{Preparation and characterization of lipid-stabilized contrast formulation}

We determined in preliminary studies that gadolinium concentrations in the range of $6-12 \mathrm{mg} / \mathrm{mL}$ were optimal for contrast enhancement on mucosal surfaces. Based on this concentration, four different lipid formulations were designed (Table 1) and prepared by the lyophilization-rehydration method. After rehydration, while some of the gadobenate dimeglumine molecules could be encapsulated, some could be simply adsorbed onto the liposome surfaces due to the excessive amount of negative ions of gadobenic acid. The resulting liposomes had mean particle sizes of around $200 \mathrm{~nm}$. The zeta potentials ranged from -39 to $-64 \mathrm{mV}$.

\section{Nebulization of lipid-stabilized contrast formulation}

The liposomal contrast solution was nebulized by ultrasonication. The sizes of the resulting droplets were measured using a Spraytec RTSizer based on the laser diffraction method. As shown in Figure 2A, for all the five groups, droplets with a volume mean diameter D $(4,3)$ of around 5-7 $\mu \mathrm{m}$ suitable for upper airway deposition were generated via ultrasonic nebulization. However, the size distribution was affected considerably by the lipid components. The lipid-stabilized droplets had much smaller Dv10 and Dv90 diameters than that of the control (Figure 2A), and concentrated in only one peak in the cumulative volume percent curve except for L4 (Figure 2B). The results suggest that the lipid components were helpful for generation and stabilization of small and uniform droplets during nebulization. 
Table I Particle sizes and zeta potentials of the lipid-stabilized contrast agents

\begin{tabular}{lllll}
\hline $\begin{array}{l}\text { Formulations } \\
\text { (in molar ratio) }\end{array}$ & $\begin{array}{l}\text { Total lipid concentration } \\
(\mathbf{m g} / \mathbf{m L})\end{array}$ & $\begin{array}{l}\text { SA to Gd } \\
\text { (molar ratio) }\end{array}$ & Size (nm) & $\begin{array}{l}\text { Zeta potential } \\
(\mathbf{m V})\end{array}$ \\
\hline (LI) EPC:cholesterol:SA = I:I:I & 32 & $\mathrm{I}: \mathrm{I}$ & $182.58 \pm 13.35$ & $-38.7 \pm 16.4$ \\
(L2) EPC:cholesterol:SA = 2:2:1 & 29 & $1: 2$ & $176.25 \pm 13.24$ & $-58.7 \pm 22.3$ \\
(L3) EPC:cholesterol:SA = I:1:2 & 19 & $\mathrm{I}: \mathrm{I}$ & $181.58 \pm 15.28$ & $-31.5 \pm 9.9$ \\
(L4) EPC:cholesterol = I:I & 26 & No SA & $169.42 \pm 11.30$ & $-63.4 \pm 16.3$ \\
\hline
\end{tabular}

Abbreviations: EPC, egg phosphotidylcholine; SA, sterylamine; Gd, gadolinium.

In addition, increasing the ratio of sterylamine in the lipid formulation seemed to have a further stabilization effect for the smaller droplets (Figure 2C). More droplets smaller than $2.15 \mu \mathrm{m}$ were observed for formulation L1 (33\% sterylamine) compared with formulation L2 (20\% sterylamine). Formulation L3 with 50\% sterylamine also produced more smaller droplets after nebulization considering that the total lipids ( $25 \mathrm{mg} / \mathrm{mL}$ ) were less in the formulation. In comparison, the L4 formulation free of sterylamine produced larger droplets after ultrasonic nebulization and two different size peaks were detected. For the control, the droplets were generally bigger, without the stabilization effects of the lipids.

\section{Deposition of nebulized contrast formulations on lumen surface of pig trachea}

Deposition of the nebulized contrast formulations on the mucosal surface of the larynx and trachea was studied

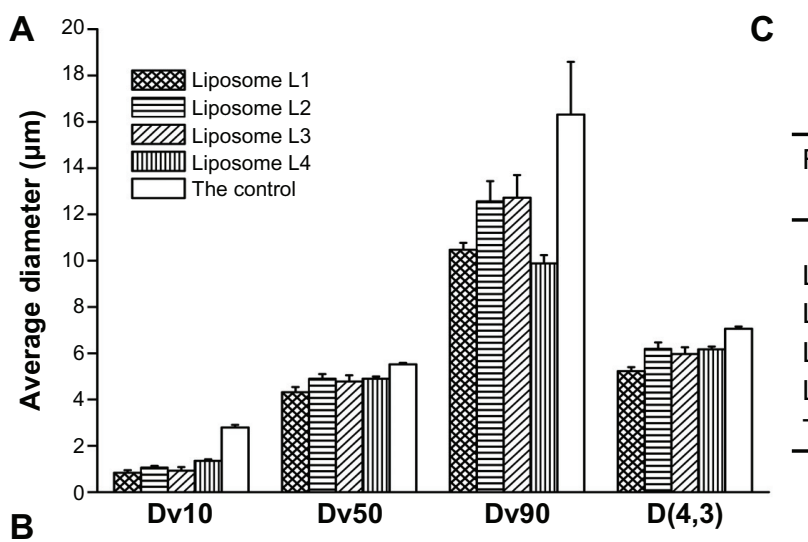

\begin{tabular}{lcc}
\hline Formulations & SA molar ratio (\%) & $\begin{array}{c}\text { Percent of droplets } \\
\text { smaller than } 2.15 \mu \mathrm{m}(\%)\end{array}$ \\
\hline & & 27.40 \\
L1 & 33 & 21.57 \\
L2 & 20 & 23.33 \\
L3 & 50 & 16.03 \\
L4 & 0 & 5.49 \\
The control & 0 & \\
\hline
\end{tabular}
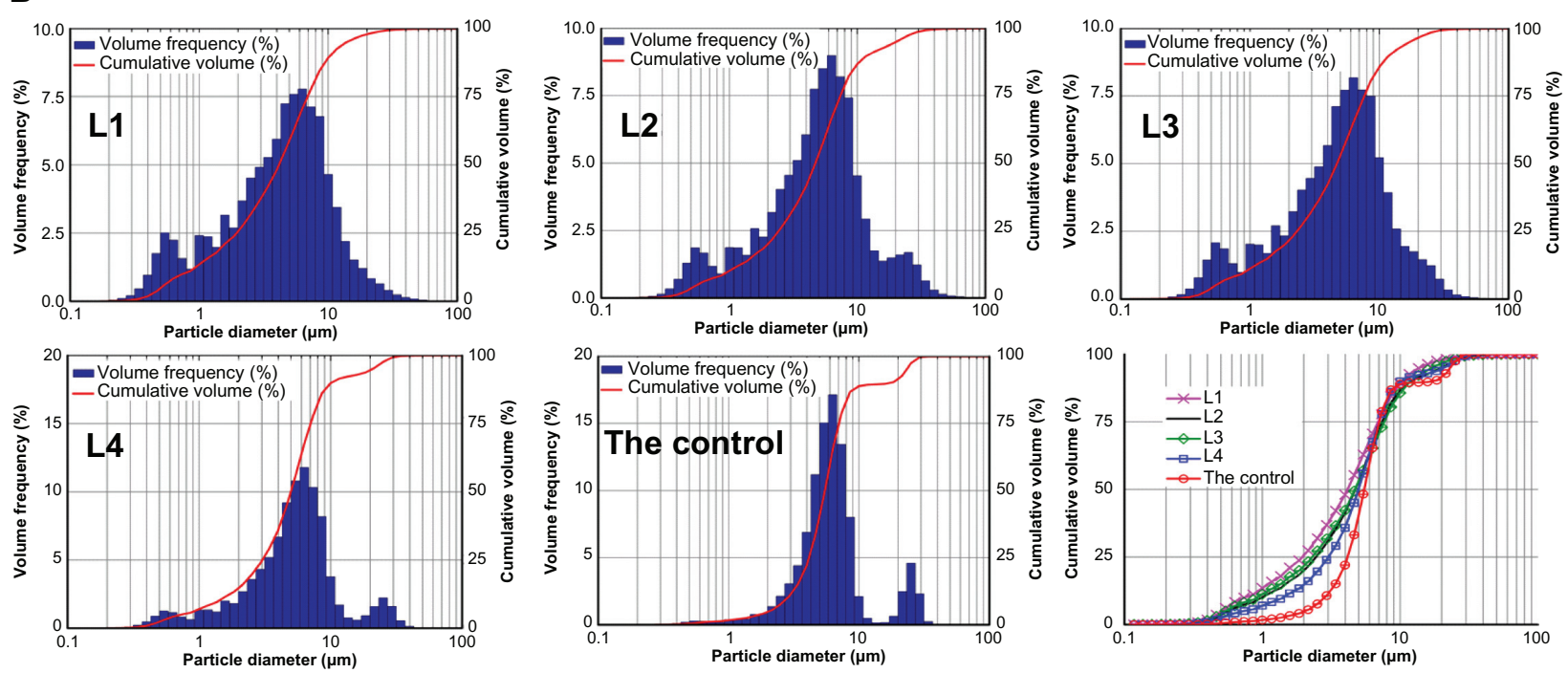

Figure 2 Average diameter (A), diameter distribution (B), and percentage of small droplets (C) of lipid-stabilized droplets and the control after ultrasonic nebulization. In B, the blue columns represent the volume frequency percentage and the red curve represents the cumulative volume percentage; in the cumulative volume overlay graph, pink indicates LI, black indicates L2, green indicates L3, blue indicates L4 and red indicates the control. 
by measuring gadolinium concentrations at different sampling sites using inductively coupled plasma atomic emission spectroscopy. As shown in Figure 3, the addition of sterylamine to the formulation greatly improved the deposition of gadolinium at the top luminal surfaces. Gadolinium concentrations from L1, L2, and L3 reached on the top half of the trachea lumen were significantly higher than that from L4 and the control. At the same time, more lipid-stabilized droplets successfully distributed to the distal segments. As a comparison, the droplets of the control and L4 formulations accumulated mainly in the bottom parts of the tracheas in the proximal segment just adjacent to the larynx, and gadolinium concentrations decreased significantly with distance. Of the four lipid-stabilized contrast agents, the L1 formulation showed the most even deposition on the tracheal mucosal surface. It was therefore used in the MRI study.

\section{MRI study of larynx and tracheal lumen surface}

The lipid-stabilized gadobenate dimeglumine formulation, L1, was nebulized and delivered to the freshly explanted pig trachea segments. The mucosal surface visualization effect was evaluated. In imaging sequence screening, the T1 weighted-spectral presaturation inversion recovery sequence showed the best visualization effect and was used in the MRI studies. As shown in Figure 4, even contrast enhancement on the laryngeal and tracheal mucosal surface was achieved with the lipid-stabilized contrast formulation. The mucosal surfaces were effectively "lightened up" with an even coating of gadolinium and were very clearly visualized. The bright signals along the lumen surface in both the larynx and trachea was generally uniform, as shown in the transverse images of different layers in successive scanning (Figure 5). In comparison, only parts of the larynx and tracheas (especially the areas in the bottom half of the lumens) were effectively contrasted with the control (Figure 4B1 and B2).

The mucosal surface visualization effect of the lipidstabilized contrast formulation was further evaluated in the three diseased tissue models (Figure 6A and B). With the nebulized lipid-stabilized contrast formulation, the small intraluminal protrusion and the submucosal nodule were clearly depicted with bright signals. In Figure 6C, one small crater (as pointed out by the arrow) on the luminal surface was also clearly visualized.

\section{Discussion}

MRI has been proven to be very useful in the diagnosis of laryngeal and tracheal tumors because of excellent soft tissue contrast and high sensitivity to cartilaginous invasion. However, due to the very low proton density and large

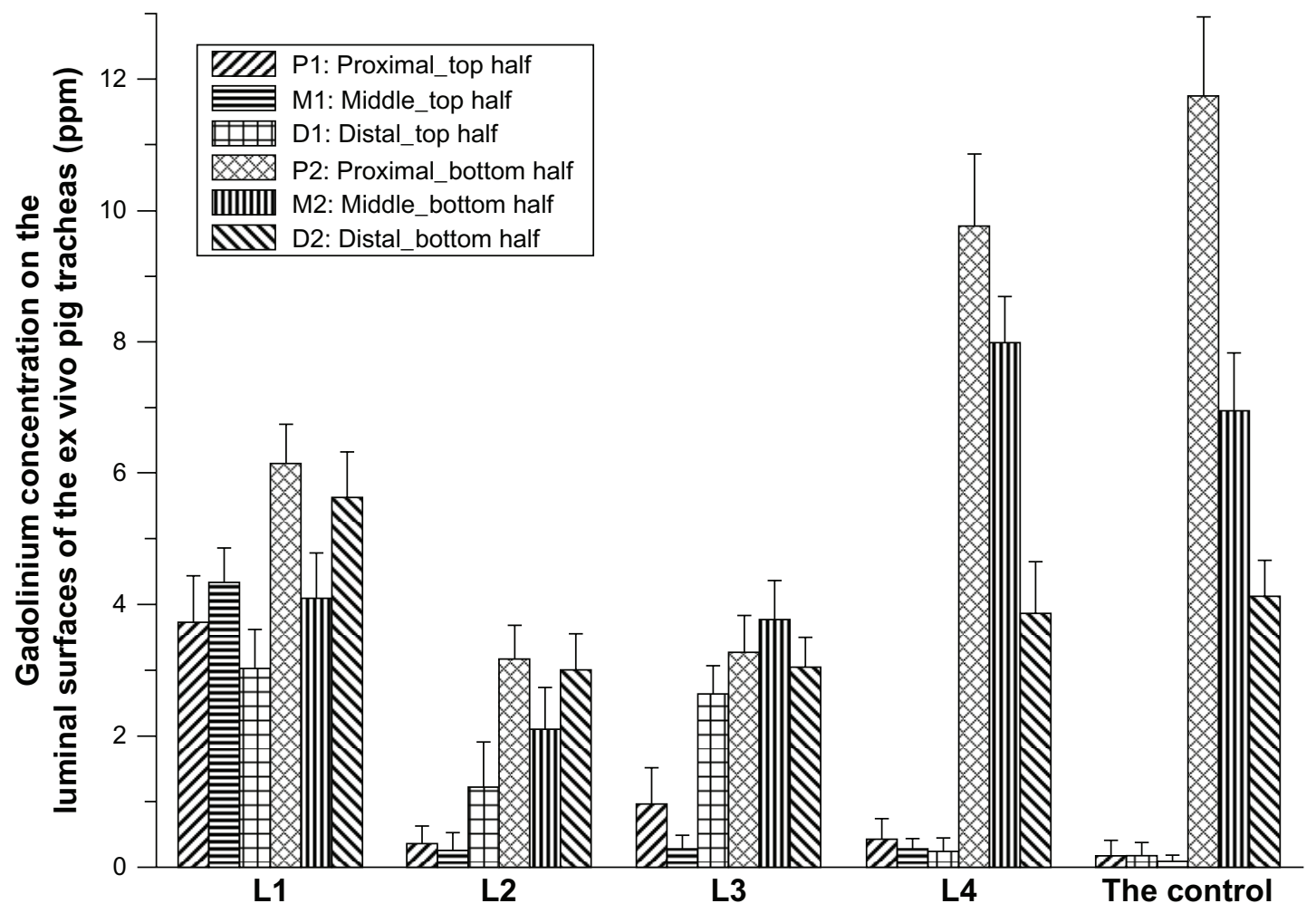

Figure 3 Gadolinium concentrations at different sites on pig tracheal luminal surfaces after nebulization delivery of lipid-stabilized contrast agent and the control. 

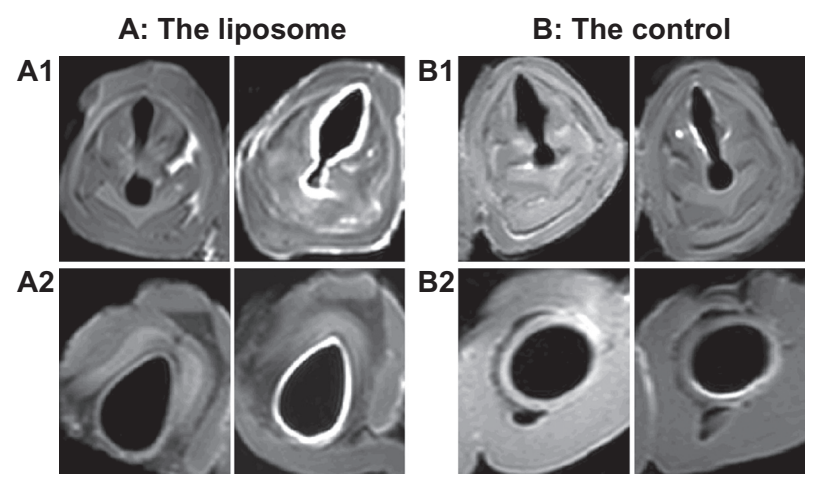

Figure 4 Transverse magnetic resonance images of ex vivo pig laryngeal ( $A$ I and $B \mathbf{I}$ ) and tracheal (A2 and B2) segments before and after nebulization delivery of lipidstabilized contrast agent and the control. Left images, before nebulization; right images, after nebulization.

air-soft tissue interfaces in the airway, visualization of the mucosal surfaces by MRI is not satisfactory. Small foci or alternations on the mucosal surface are reported to be hard to detect or invisible. ${ }^{2}$ Because most malignant laryngeal and tracheal tumors occur in the epithelium with alterations on the mucosal surface, we tried to improve the sensitivity of MRI for early detection of mucosal lesions by direct clear visualization of the mucosal surfaces. In order to enhance the signals on the mucosal surface, we designed a lipid-stabilized formulation for a gadolinium contrast agent. The nebulized contrast formulation could coat evenly on the mucosal surface, and light up the surface for MRI. Some earlier studies have used aerosolized gadolinium solutions to evaluate pulmonary ventilation, ${ }^{9,20-22}$ but there has been no report on mucosal coating contrast agents specifically designed to visualize the laryngeal and tracheal surfaces for MRI.

In order to achieve an even coating effect on the laryngeal and tracheal mucosal surfaces for imaging, we selected lipids as the stabilization and mucoadhesion agent. Lipids
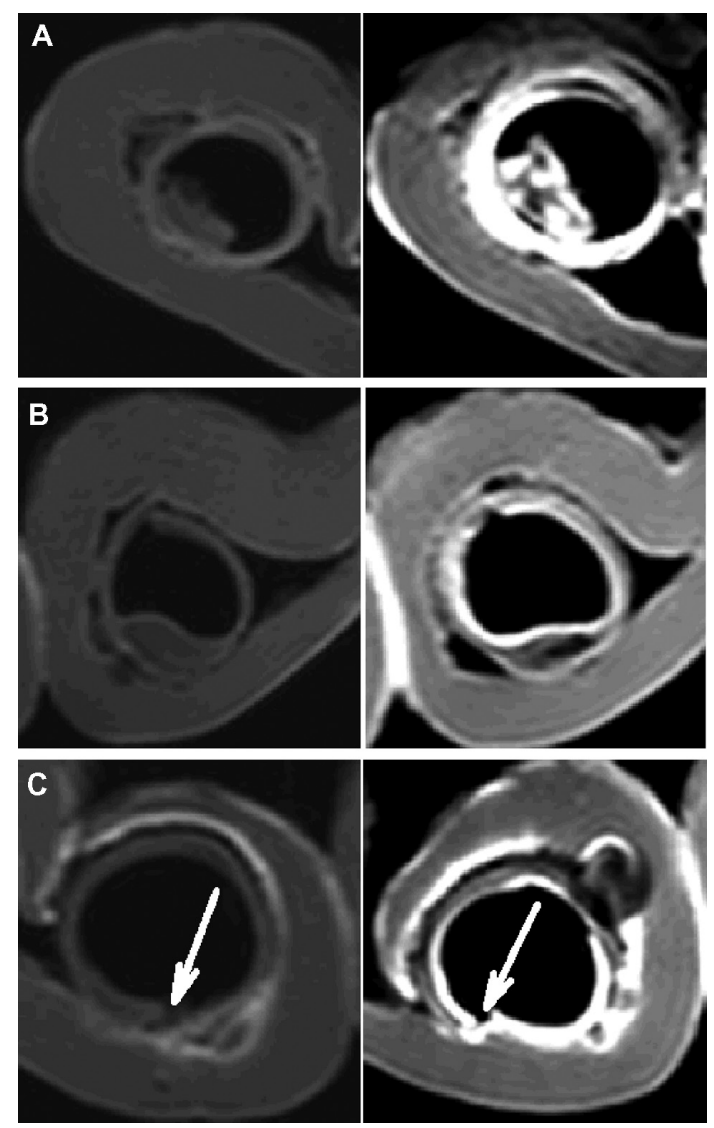

Figure 6 Transverse magnetic resonance images of three diseased tissue models before (left column) and after (right column) contrast enhancement by the nebulized lipid-stabilized contrast formulation. (A) Intraluminal protrusion, (B) submucosal nodule, and (C) submucosal crater.

and liposomes have been widely used for pulmonary delivery of therapeutic proteins or chemical compounds..$^{23-25}$ In recent years, cationic liposome systems were developed for improved gene delivery to airway mucosal surfaces. . $82,26,27^{2}$ Compared with other materials, such as polymers, lipids have good safety and biocompatibility. Therefore, we used egg
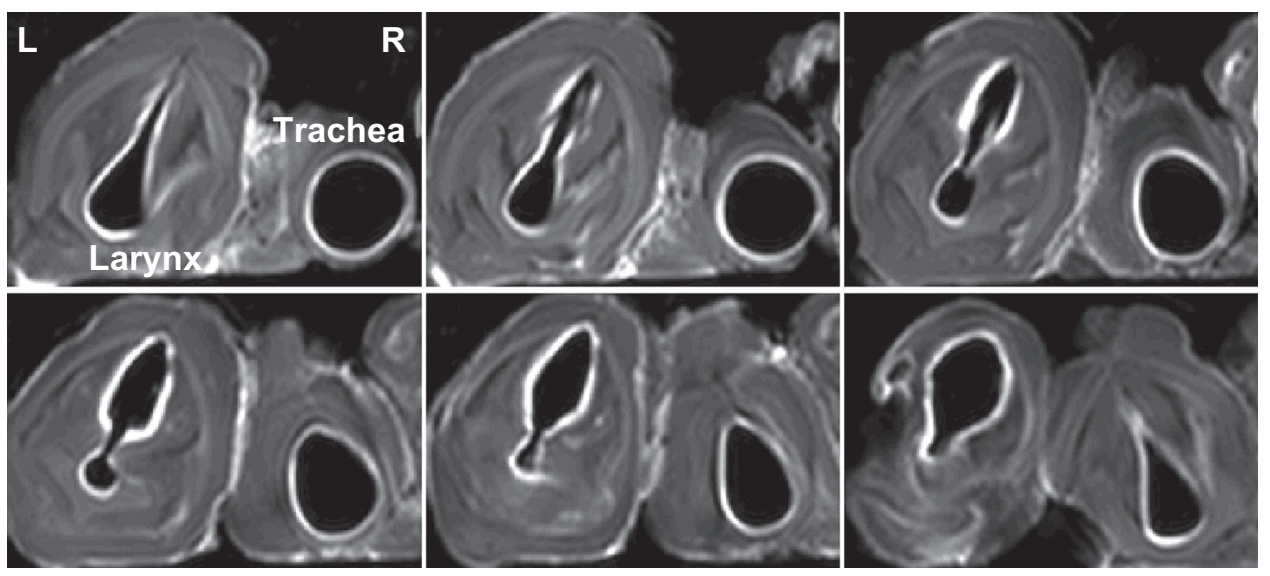

Figure 5 Transverse magnetic resonance images of ex vivo pig laryngeal and tracheal lumen in successive scanning layers after nebulization delivery of the lipid-stabilized contrast agent. In each image, the left is the larynx, and the right is the tracheal lumen. 
phosphatidylcholine as the main lipid component for formation of fluid phase membrane vesicles, and cholesterol in high molar ratios ranging from $25 \%$ to $50 \%$ was included.

We included sterylamine in the formulations to improve the mucoadhesive effect of the droplets. The mucosal surface is covered by a thin layer of mucus, with major components of mucin glycoproteins, lipids, inorganic salts, and water. ${ }^{28}$ Because most mucin glycoproteins have a strongly negatively charged surface due to a high sialic acid and sulfate content, ${ }^{29}$ various positively charged materials could be used to enhance the electric interactions with the mucosal surface ${ }^{28}$ and help the vehicles to adhere to the mucosal surface effectively. In our study, sterylamine was positively charged at neutral $\mathrm{pH}$, and its single fatty acid chain could easily insert into the phospholipid bilayers.

The gadolinium agent was loaded into the lipid vehicles using the lyophilization-rehydration method, and particles with a mean diameter of about $200 \mathrm{~nm}$ were generated. Interestingly, the particles were negatively charged, with zeta potentials ranging from $-39 \mathrm{mV}$ to $-64 \mathrm{mV}$. We believe this to be because of the excessive negative charges in the gadobenate dimeglumine molecules adsorbed on the vehicle surfaces. As a rather toxic ion, gadolinium in free form $\left(\mathrm{Gd}^{3+}\right)$ must be chelated to form a stable complex for in vivo imaging applications. For gadobenate dimeglumine, the gadolinium ion is chelated with 4-carboxy-5,8,11-tris(carboxymethyl)1-phenyl-2-oxa-5,8,11-triazatridecan-13-oic acid to form gadobenic acid $\left[\mathrm{Gd}(\text { bopta })\left(\mathrm{H}_{2} \mathrm{O}\right)\right]^{2-}{ }^{2}{ }^{30}$ The two extra negative charges on each gadobenic acid molecule may interact with the positively charged amine of sterylamine and create a negatively charged surface. Therefore, the particles had negative zeta potentials.

In addition to mucoadhesive properties, the droplets should also be of a suitable size range for effective deposition on the laryngeal and tracheal mucosal surface. Deposition of nebulized droplets in the respiratory tract was significantly affected by their sizes. Droplets larger than $5 \mu \mathrm{m}$ would impact on the larynx and pharynx after inhalation. Droplets with diameters of $0.5-5 \mu \mathrm{m}$ would sediment in the airway, and droplets smaller than $0.5 \mu \mathrm{m}$ would be in Brownian motion and settle very slowly in the airway lumen. ${ }^{19}$ In our study, we characterized the sizes of nebulized droplets stabilized with various lipid formulations using the laser diffraction method. This method has been used in aerosol formulation studies and proven to be a very fast and highly reliable technique. ${ }^{31}$ Compared with the more commonly used cascade impactor analysis, the size distributions measured by the laser diffraction method are independent of the flow rate and could be automatically recorded as a function of inhalation time. ${ }^{31}$ As shown in Figure 2A, the mean volume diameter of the droplets was around 4-6 $\mu \mathrm{m}$. As expected, the amphiphilic lipid components effectively helped to generate droplets in a narrow size distribution.

Deposition of the nebulized droplets on the mucosal surface of the trachea was studied by measuring gadolinium concentrations at different sites in the lumen. In order to evaluate the mucoadhesive effect of the droplets, we positioned the pig tracheal segments horizontally during nebulization delivery, then cut them open vertically to compare deposits on the top and bottom segments. The delivery time was set to be two minutes to ensure effective coating without droplet coagulation or flowing on the surface. As shown in Figure 3, in all the groups, gadolinium concentrations in the bottom half of the mucosal surface of the trachea were always higher than those at the top half, in part due to the gravity effect. However, the three lipid formulations containing sterylamine (L1, L2, and L3) effectively improved adsorption of droplets to the top half of the mucosal surface. According to Smart, ${ }^{28}$ the mucoadhesion process would include two steps, ie, contact and consolidation. The droplets or particles would firstly come into intimate contact with the mucosal surface, and then a strengthened adhesive joint would form through various physicochemical interactions, leading to a prolonged adhesion effect. On this basis, we believe that the improved deposition profiles of the three lipid-stabilized droplets may be attributable to the amphiphilic properties of the lipids and the positive surface charges on the cationic lipid, sterylamine. The presence of amphiphilic lipids would improve contact of the droplets with the mucosal surface by decreasing the surface energy of the droplets. The adhesive process was further consolidated by the charge interactions between the positively charged sterylamine and negatively charged mucosal surface.

We also believe that the smaller droplets in the three formulations helped their mucoadhesion. As shown in Figure 2C, the addition of sterylamine resulted in smaller droplets with a diameter of less than $2.15 \mu \mathrm{m}$ after nebulization. The smaller droplets with large surface area to volume ratios and higher attractive forces ${ }^{28}$ would move in the lumen with the direction of the airstream and adhere to the mucosal surface whenever the interactions were strong enough. We noticed that in the proximal parts of the lumen where the gas flow changed dramatically due to the cross-sectional diameter moving from the larynx to the trachea, ${ }^{32}$ the smaller particles seemed to have more chance to interact with the mucosal surface, and better deposition was observed for L2 and L3. For the same 
reason, in the distal part at the end of the tracheal segments, better deposition by L 2 and L3 was also achieved. Therefore, we think the improved deposition effect of L1 was not merely due to the presence of sterylamine in the formulation. The lipid ratios and total amounts of lipid also played an important role in the generation, stabilization, and mucoadhesion of the droplets via nebulization.

For the formulations free of sterylamine, the droplets mainly deposited in the bottom half of the mucosal surfaces of the trachea, and gadolinium concentrations were much higher on the proximal surface. This is probably because of the larger droplets in these two groups. The medium-sized droplets were more likely to deposit onto the middle and distal areas along the tracheal lumen. It should be noticed that the amount of gadolinium delivered with the L4 formulation and the control was much higher than that with the other three lipid groups. This could be explained by the higher nebulization efficiency of L4 and the control due to their relatively low viscosities in the five groups. There were more droplets obtained for the group with low viscosity (the droplet volume concentrations for L1, L4, and the control were 7.4, 25.3, and 77.0 ppm, respectively).

In the MRI study, the mucosal surface of freshly explanted pig laryngeal and tracheal segments was very clearly visualized using the lipid-stabilized contrast agent. The mucosal surface was outlined smoothly and evenly with bright signals. Considering the complex anatomic structures in the larynx and the complicated gas flows in the upper airway, the even coating and contrast enhancement with the nebulized agent was a significant improvement. We further evaluated the visualization effects for three common airway lesions. The shapes and locations of all the representative tissue abnormalities were successfully depicted. These preliminary results suggest that a lipid-stabilized contrast agent would be beneficial for improvement of MRI in early diagnosis of diseases of the larynx and trachea.

\section{Conclusion}

In this study, a liposomal gadobenate dimeglumine contrast formulation was successfully developed for clear MRI visualization of the mucosal surface on the larynx and trachea via nebulization. Various cationic liposome formulations were compared for their stabilization effects on gadobenate dimeglumine-containing droplets. Liposomes composed of egg phosphotidylcholine, cholesterol, and stearylamine in a molar ratio of $1: 1: 1$ were found to have the most efficient nebulization effect, and had the most narrowly distributed droplet sizes. The nebulized droplets were of mean diameter
4-6 $\mu \mathrm{m}$ after ultrasonic nebulization. They evenly coated on the laryngeal and tracheal lumen surfaces and produced significant contrast enhancement along the mucosal surface. Such contrast enhancement enabled clearer visualization of several disease states, such as intraluminal protrusions, submucosal nodules, and craters in ex vivo pig laryngeal and tracheal segments. The liposomal MRI contrast formulation may be useful for improving visualization of the mucosal surface and early diagnosis of diseases originating from the mucosal surfaces of the larynx and trachea.

\section{Acknowledgments}

The authors are grateful to Shi $\mathrm{Xu}$ from the Analytical Center of Shanghai Jiao Tong University for performing the inductively coupled plasma atomic emission spectroscopy measurements, and to Tom Qin of Malvern, China, for his assistance with measurement of nebulized droplet sizes.

\section{Disclosure}

The authors report no conflicts of interest in this work.

\section{References}

1. Becker M, Burkhardt K, Dulguerov P, Allal A. Imaging of the larynx and hypopharynx. Eur J Radiol. 2008;66(3):460-479.

2. Hermans R. Staging of laryngeal and hypopharyngeal cancer: value of imaging studies. Eur Radiol. 2006;16(11):2386-2400.

3. Mathisen D. Primary tracheal tumor management. Surg Oncol Clin NAm. 1999;8(2):307.

4. Connor S. Laryngeal cancer: how does the radiologist help? Cancer Imaging. 2007;7(1):93.

5. Deliyski DD, Hillman RE. State of the art laryngeal imaging: research and clinical implications. Curr Opin Otolaryngol Head Neck Surg. 2010;18(3):147-152.

6. Kauczor HU, Kreitner KF. MRI of the pulmonary parenchyma. Eur Radiol. 1999;9(9):1755-1764.

7. Gast KK, Eberle B, Schmiedeskamp J, Kauczor HU. Magnetic resonance imaging using hyperpolarized He-3-gas. Acad Radiol. 2003;10(10):1119-1131.

8. Patz S, Hersman FW, Muradian I, et al. Hyperpolarized Xe-129 MRI: a viable functional lung imaging modality? Eur J Radiol. 2007;64(3):335-344.

9. Haage P, Karaagac S, Spuntrup E, Truong HT, Schmidt T, Gunther RW. Feasibility of pulmonary ventilation visualization with aerosolized magnetic resonance contrast media. Invest Radiol. 2005;40(2):85-88.

10. Vyas SP, Pawar D, Goyal AK, et al. Evaluation of mucoadhesive PLGA microparticles for nasal immunization. AAPS J. 2010;12(2):130-137.

11. Pongjanyakul T, Suksri H. Alginate-magnesium aluminum silicate films for buccal delivery of nicotine. Colloids Surf B Biointerfaces. 2009;74(1):103-113.

12. Erjavec V, Pavlica Z, Sentjurc M, Petelin M. In vivo study of liposomes as drug carriers to oral mucosa using EPR oximetry. Int $J$ Pharm. 2006;307(1):1-8.

13. Majumdar DK, Thakral NK, Ray AR, Bar-Shalom D, Eriksson AH. The quest for targeted delivery in colon cancer: mucoadhesive valdecoxib microspheres. Int J Nanomed. 2011;6:1057-1068.

14. Alonso MJ, Oyarzun-Ampuero FA, Brea J, Loza MI, Torres D. Chitosanhyaluronic acid nanoparticles loaded with heparin for the treatment of asthma. Int J Pharm. 2009;381(2):122-129. 
15. Antimisiaris SG, Zaru M, Manca ML, Fadda AM. Chitosan-coated liposomes for delivery to lungs by nebulisation. Colloids Surf B Biointerfaces. 2009;71(1):88-95.

16. Mortazavi SA, Mehravaran N, Moghimi H. The influence of various mucoadhesive polymers on in vitro performance of the resulting artificial saliva pump spray formulations. Iran J Pharm Res. 2009;8(1):3-13.

17. Gattani SG, Savaliya PJ, Belgamwar VS. Floating-mucoadhesive beads of clarithromycin for the treatment of Helicobacter pylori infection. Chem Pharm Bull (Tokyo). 2010;58(6):782-787.

18. Pillai R, Petrak K, Blezinger P, et al. Ultrasonic nebulization of cationic lipid-based gene delivery systems for airway administration. Pharm Res. 1998;15(11):1743-1747.

19. Amighi K, Pilcer G. Formulation strategy and use of excipients in pulmonary drug delivery. Int J Pharm. 2010;392(1-2):1-19.

20. Misselwitz B, Muhler A, Heinzelmann I, Bock JC, Weinmann HJ. Magnetic resonance imaging of pulmonary ventilation - initial experiences with a gadolinium-DTPA-based aerosol. Invest Radiol. 1997;32(12):797-801.

21. Haage P, Adam G, Karaagac S, et al. Mechanical delivery of aerosolized gadolinium-DTPA for pulmonary ventilation assessment in MR imaging. Invest Radiol. 2001;36(4):240-243.

22. Haage P, Karaagac S, Spuntrup E, Adam G, Gunther RW. MR imaging of lung ventilation with aerosolized gadolinium-chelates. Rofo. 2003; 175(2):187-193. German.

23. Antimisiaris SG, Zaru M, Mourtas S, Klepetsanis P, Fadda AM. Liposomes for drug delivery to the lungs by nebulization. Eur J Pharm Biopharm. 2007;67(3):655-666.
24. Huang YY, Wang CH. Pulmonary delivery of insulin by liposomal carriers. J Control Release. 2006;113(1):9-14.

25. Lu DM, Hickey AJ. Liposomal dry powders as aerosols for pulmonary delivery of proteins. AAPS Pharm Sci Tech. 2005;6(4):E641-E648.

26. Kedar E, Even-Or O, Joseph A, et al. A new intranasal influenza vaccine based on a novel polycationic lipid-ceramide carbamoyl-spermine (CCS). II. Studies in mice and ferrets and mechanism of adjuvanticity. Vaccine. 2011;29(13):2474-2486.

27. Liu DZ, Tseng LP, Chiou CJ, et al. Evaluation of encapsulated newcastle disease virus liposomes using various phospholipids administered to improve chicken humoral immunity. J Biomed Mater Res B. 2009; 91B(2):621-625.

28. Smart JD. The basics and underlying mechanisms of mucoadhesion. Adv Drug Deliv Rev. 2005;57(11):1556-1568.

29. Hanes J, Lai SK, Wang YY, Wirtz D. Micro- and macrorheology of mucus. Adv Drug Deliv Rev. 2009;61(2):86-100.

30. Werner EJ, Datta A, Jocher CJ, Raymond KN. High relaxivity MRI contrast agents: where coordination chemistry meets medical imaging. Angew Chem Int Ed. 2008;47(45):8568-8580.

31. de Boer AH, Gjaltema D, Hagedoorn P, Frijlink HW. Characterization of inhalation aerosols: a critical evaluation of cascade impactor analysis and laser diffraction technique. Int J Pharm. 2002;249(1-2): 219-231.

32. Ehtezazi T, Southern KW, Allanson D, Jenkinson I, O’Callaghan C. Suitability of the upper airway models obtained from MRI studies in simulating drug lung deposition from inhalers. Pharm Res. 2005;22(1) $166-170$.
International Journal of Nanomedicine

\section{Publish your work in this journal}

The International Journal of Nanomedicine is an international, peerreviewed journal focusing on the application of nanotechnology in diagnostics, therapeutics, and drug delivery systems throughout the biomedical field. This journal is indexed on PubMed Central,

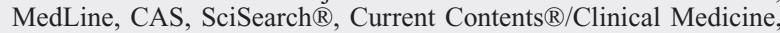

\section{Dovepress}

Journal Citation Reports/Science Edition, EMBase, Scopus and the Elsevier Bibliographic databases. The manuscript management system is completely online and includes a very quick and fair peer-review system, which is all easy to use. Visit http://www.dovepress.com/ testimonials.php to read real quotes from published authors 